\title{
Erregen der Wehenthätigkeit durch intrauterine Injection von Glycerin.
}

\author{
Von \\ Dr, med. C. Pelzer, \\ Assistenzarzt an der Provinzial-Hebammenlehranstalt zu Köln.
}

(Nach einem Vortrage, gehalten in der Gesellschaft für Geburtshülfe und Gynäkologie zu Köln a. Rh.)

Es sind im Laufe der Zeit viele Mittel angegeben worden, um die Wehenthätigkeit zum Zwecke der künstlichen Frühgeburt anzuregen. Die meisten davon hat man als unwirksam oder sogar gef ährlich wieder verlassen, und nur einige wenige sind noch heute in Gebrauch.

Wenn wir von den ganz unzuverlässigen medicamentösen und äusseren Mitteln absehen, so wollen wir hier unser Augenmerk nur auf solche Mittel lenken, die Wehen zu erregen suchen, indem sie direct von Scheide oder Uterus aus einwirken.

Von den verschiedenen Methoden seien hier angeführt: die heisse Uterusdouche, die Dilatation der Cervix, die Katheterisation des Uterus, der Eihautstich und die intrauterine Injection. Als sicher wirkend sind von diesen Mitteln nur die drei letzten anerkannt worden.

Was die Uterusdouche angeht, so ist sie, abgesehen von ihrer Unbequemlichkeit für Schwangere und Arzt, nur unsicher wirkend und zuweilen sogar gefährlich. So hat Litzmann einen Todesfall durch Luftembolie bei Anwendung derselben zu verzeichnen.

Die Dilatation der Cervix durch Pressschwämme und Laminariastifte ist auch nicht ungefährlich. Indem dieselben nämlich die Gebärmuttersecrete in sich aufnehmen, kann leicht Zersetzung und Infection eintreten. Mehrere Todesfälle haben denn auch thatsächlich dazu beigetragen, dass diese Methode heute wieder ziemlich verlassen ist.

$\mathrm{Zu}$ den eigentlich sicheren Mitteln, die künstliche Frühgeburt herbeizufiihren, sind die Katheterisation des Uterus, der Eihautstich und die intrauterine Injection zu rechnen. 
Die Katheterisation hat den Nachtheil des verzögerten Weheneintrittes und bei dem oft tagelangen Liegenlassen des Bougies die Gefahr der Infection. Von 9 künstlichen Frühgeburten, die in hiesiger Anstalt durch Einlegen eines Bougies eingeleitet wurden, begannen in 2 Fällen die Wehen erst nach 2 Tagen, $1 \mathrm{Mal}$ erst nach 4 Tagen. Bei letzterem wurden wiederholt Schüttelfröste beobachtet. Bei 2 traten die Wehen zwar bald nach dem Einlegen ein, sistirten dann aber vollständig, so dass es eines mehrmaligen Einführens bedurfte. Zwei Mal liess diese Methode überhaupt im Stiche, so dass die Wehen erst nach dem spontanen Blasensprunge, ein anderes Mal nach stundenlangem Kneten des Leibes einsetzten. Beide Patientinnen hatten Schüttelfrost bei einer Temperatur von $40-40,5^{\circ}$.

Nur 2 Fälle sind zu verzeichnen, wo die Wehenthätigkeit 1-3 Stunden nach dem Einlegen des Bougies begann, die Wehen anhielten, die Geburt förderten und das Kind nach 30-40 Stunden geboren wurde. Von den 9 Kindern kam 1 todt, $\mathbf{3}$ andere todtfaul zur Welt. Bei diesen hatten die Mütter Schüttelfrost gehabt.

Der Eihautstich zur Einleitung der künstlichen Frühgeburt ist zwar sehr sicher wirkend, hat aber alle Nachtheile des vorzeitig abgeflossenen Fruchtwassers, d. i. verzögerte Geburt durch unregelmässige Wehen, die wieder das Leben des Kindes gefährden, als auch die Gefahr der Infection.

In 2 von unseren Fällen begannen die Wehen erst 26 Stunden nach dem Eihautstiche. Die Geburt wurde im ersten 12 Stunden später bei fünfmarkstückgrossem Muttermunde durch Wendung und Extraction beendet. Das Kind war todt und während der Geburt trat Schüttelfrost auf, bei einer Temperatur von $40,4^{\circ}$

Im zweiten Falle erfolgte die Geburt weitere 27 Stunden nach Beginn der Wehen spontan. Ein anderes Mal wurde nach viertägigen schwachen Wehen bei fünfmarkstückgrossem Muttermunde das Kind gewendet und darauf todt extrahirt.

Aus den angeführten Thatsachen 'geht hervor, dass sowohl die Katheterisation des Uterus, als auch der Eihautstich doch noch Manches zum Wohle für Mutter und Kind zu wünschen übrig lassen.

Die idealste Methode zur Einleitung der künstlichen Frühgeburt wäre natürlich die, welche bei baldigen guten Wehen die Blase möglichst schonte, die Geburt förderte und die Gefahr der 
Infection auf ein Minimum herabsetzte. Zum grossen Theile nun sind diese Anforderungen durch die intraterine Injection erreicht worden. Wenn dieser Methode ausser der Infection noch die Gefahr des Lufteintrittes in die Uterusvenen anhaftet, so ist doch letzteres durch geeignete Vorsichtsmaassregeln zu vermeiden.

Cohen leitete die künstliche Frühgeburt durch Einspritzen von Theerwasser zwischen Eihäute und Uterus-Innenfläche ein. In der Neuen Zeitschrift für Geburtskunde 1846, Bd. XXI, S. 116 theilt er einen Fall mit, wo in einem Zeitraume von fünf Stunden zwei Mal injicirt wurde. Acht Stunden später traten kräftige Wehen auf, wodurch das Kind, welches in Fusslage lag, nach elf Stunden bis zu den Schultern geboren und darauf der Kopf durch Kunsthülfe entwickelt wurde. Allerdings trat einmal während der Geburt Schüttelfrost auf. Das Wochenbett verlief jedoch normal und das Kind gedieh vortrefflich.

Litzmann benutzte zur Injection Wasser von $40^{\circ}$, das er in Mengen von $500 \mathrm{~g}$ injicirte. Nachdem jedoch verschiedene Fälle von Tod der Mutter durch Luftembolie publicirt worden waren, hat man diese Methode fast vollständig wieder verlassen. In diesem Archive, Bd. II, S. 169 hat Litzmann ausserdem einen Fall veröffentlicht, wo 2 Stunden nach der Injection heftiger Schüttelfrost eintrat, nach 36 Stunden ein todtes Kind geboren wurde und die Mutter 17 Stunden nach ihrer Entbindung an Sepsis zu Grunde ging.

Bei diesen Thatsachen nun würde es sich darum handeln, bei allen Vortheilen der intrauterinen Injection das Einbringen von Luft sowohl, als auch von ansteckenden Stoffen in das Uteruscavum möglichst zu vermeiden. Ersteres ist bei der nöthigen Vorsicht wohl zu umgehen. Was die Infection betrifft, so käme es darauf an, womöglich eine desinficirende Flüssigkeit selbst einzuspritzen, die aber auch wiederum nicht zu sehr reizt.

Im Juli 1890 nun ist in hiesiger Anstalt zuerst der Versuch gemacht worden, Glycerin zwischen Eihäute und Uteruswand einzuspritzen. Glycerin ist an und für sich ein Antisepticum. Wie man sich seine weitere Wirkung zur Erregung der Wehenthätigkeit zu denken hat, werde ich später ausführen. Zur Injection bedienten wir uns anfangs einer Ballonspritze mit langem Ansatzrohre von Hartgummi, welches an der Spitze nach Art eines Mercier'schen Katheters gebogen war. Nachdem die Spritze, von beiläufig $50 \mathrm{ccm}$ Inhalt, mit Glycerin gefüllt war, wurde die 
Luft im Rohre selbst durch leichtes Zusammendrücken des Ballons ausgetrieben und dann die Spritze zwischen Eihäute und Uterus-Innenfläche so eingeführt, dass die Spitze selbst der hinteren Uteruswand, die Rundung des Rohres der Fruchtblase zugewendet war. Auf diese Weise wird eine Verletzung der Eihäute, sowie das directe Wiederabfliessen des Glycerins möglichst vermieden. Hierbei ist noch die Verlängerung der Cervix in der Schwangerschaft zu beachten, so dass also die Spritze möglichst weit, bis über den inneren Muttermund, hineingeführt werden muss. Erst nachdem dies geschehen, wurde der Inhalt des Ballons in die Gebärmutter entleert. Zwei Spritzen genügten immer zur Erregung deutlicher Wehen. Um nun den Lufteintritt in die Uterusvenen fast mit absoluter Sicherheit zu vermeideu, bedienen wir uns jetzt einer Wundspritze mit gut schliessendem Kolben von $150 \mathrm{~g}$ Inhalt. Dieselbe wird mit Glycerin gefüllt und durch einen Gummischlauch mit einem Mercier'schen Katheter verbunden. Die Luft wird aus dem Katheter durch Einspritzen von Glycerin aus der Wundspritze ausgetrieben. Jetzt erst wird der Katheter unter beständigem Ausfliessen von Glyeerin an der hinteren Gebärmutterwand so weit wie möglich emporgeführt und dann die ganze Spritze entleert, so dass nunmehr ca. $100 \mathrm{ccm}$ Glycerin injicirt sind. Um ein Rückfliessen zu verhuiten, wird die Frau mit erhöhtem Steisse in Knie-Ellenbogen- oder in Sims' Seitenlage gelegt.

Es sei mir gestattet, an der Hand einiger Krankengeschichten die Wirkung der intrauterinen Injection von Glycerin näher zu beleuchten :

1. Fall. Allgemein verengtes rhachitisches Becken. Bei diesem wurde mit der Spitze der Spritze, da die Methode noch nicht so weit ausprobirt war wie bei den späteren Fällen, die Fruchtblase verletzt und somit die Frühgeburt unfreiwillig durch den Eihautstich eingeleitet. Das Kind wurde zwei Tage später bei fünfmarkstückgrossem Muttermunde durch die Zange entwickelt und gedieh vortrefflich. Wochenbett normal.

2. F all. Frau D., 33 Jahre alt, VIII para. Deutliche Zeichen von früherer Rhachitis. Das erste Kind, durch Kunsthülfe entwickelt, kam todt zur Welt. Die zweite Schwangerschaft endete mit Abort. Fünf Mal wurde die künstliche Frühgeburt eingeleitet, von welchen Kindern nur eines am Leben blieb. Die Frau war jetzt zum achten Male, und zwar in der 32. Woche, schwanger. 
Sie kam zur Einleitung der künstlichen Frühgeburt in die Anstalt. Letzte Periode am 1. November 1890. Das Becken war ein allgemein verengtes, plattes, rhachitisches. Die Maasse waren: Spinae 26, Cristae 25,5, Trochanteren 29, D. B. 16,5, Conjugata diagonalis $7,5 \mathrm{~cm}$. Die Frucht lag in 1. Schädellage, die Herztöne deutlich zu hören.

Am 12. Juni, 6 Uhr Nachmittags intrauterine Injection von etwa $00 \mathrm{ccm}$ Glycerin. Fine halbe Stunde später Beginn der Wehen, die sich in halbstündigen $\mathrm{Z}$ wischenpausen wiederholten. Am 13. Juni, 10 Uhr Vormittags wurden die Wehen häufiger und regelmässig; $1 / 210$ Uhr Abends Muttermund vollständig erweitert; Blasensprung. Weil der Kopf sich noch nicht eingestellt, die Herztöne aber schwach wurden, Wendung und Extraction. Das Kind starb 1/4 Stunde nach der Geburt. Die Mutter nach normalem Wochenbett am 12. Tage entlassen.

3. Fall. Frau W., IV para, 26 Jahre alt. Erste Geburt und Wochenbett normal. Zweite Geburt Zange; Kind todt. Dritte Geburt Zange; Kind starb eine Stunde nach der Geburt. Jedesmal Fieber im Wochenbette. Das Becken war ein theilweise verengtes, plattes Becken. Die Maasse waren: Spinae 26, Cristae 29, Trochanteren 32, D. B. 18, Conjugata diagonalis $93 / 1 \mathrm{~cm}$. Patientin kam zur Einleitung der künstlichen Frühgeburt in die Anstalt. Letzte Periode am 27. December 1890. Die Frau war jetzt in der 36. Woche ihrer Schwangerschaft.

Am 1. September 1891, 71/2 Uhr Nachmittags Einspritzung von etwa $50 \mathrm{ccm}$ Glycerin. Wehenbeginn eine Stunde später, zuerst schwach, dann aber bald kräftig und alle fünf Minuten wiederkehrend. Am 2. September, 5 Uhr Vormittags, also 81/2 Stunden nach dem Anfange der Wehen, wurde das Kind in Glückshaube und in II. Schädellage geboren. Das Wochenbett verlief normal und Mutter und Kind wurden nach zehn Tagen gesund entlassen.

4. Fall. Frau S., 40 Jahre alt, XI para. Die acht ersten Geburten normal; bei der neunten und zehnten starke Blutung und künstliche Lösung der Nachgeburt. Letzte Periode am 15. Januar 1891. Jetzt befand sich die Frau am Ende der 32. Woche ihrer Schwangerschaft. Die letzten 14 Tage starke Blutungen. Bei ihrem Eintritt in die Anstalt mässige Blutung, dabei eine Temperatur von $39^{\circ}$. Die Untersuchung ergab: Placenta praevia lateralis. 
Am 4. September 1891, 121/4 Uhr Mittags Injection von etwa $50 \mathrm{ccm}$ Glycerin. 121/2 Uhr Nachmittags Erbrechen. $1 \frac{1}{4} \mathrm{Uhr}$ maass Patientin $40,8^{\circ}$. Zur selben Zeit, also $1 \frac{1}{2}$. Stunde nach der Injection, setzten die ersten Wehen ein. 4. September, $4 \frac{1}{2}$ Uhr Nachmittags wegen starker Blutung Wendung bei dreimarkstückgrossem Muttermunde. Um 6 Uhr Extraction des todten Kindes. Temperatur nach der Geburt 37,9 ${ }^{\circ}$. Da die Frau sehr schwach: Excitantien. Das Wochenbett verlief normal. Patientin wurde nach elf Tagen gesund entlassen.

Wodurch hat nun das Glycerin diesen günstigen Einfluss auf Erregung der Wehenthätigkeit?

Wie die früher gebräuchlichen Mittel, also Theerwasser nach Cohen und einfach 40 grädiges Wasser nach Litrmann, löst auch das immerhin unter einem ziemlichen Drucke eingespritzte Glycerin mechanisch die Eihäute in grösserer Ausdehnung von der Gebärmutterwand ab. Dabei kommt aber noch seine wasserentziehende Kraft in Betracht. Durch sie wird ein Theil des Fruchtwassers dem Eisacke entzogen und derselbe so noch mehr von der Gebärmutterwand abgehoben. Dass dem so ist, habe ich dem Auge selbst zugänglich zu machen versucht. Wenn man nämlich ein durch Abort gewonnenes vollständiges $\mathrm{Ei}$ in Glycerin legt, so wird man nach einiger Zeit bemerken, dass der Fruchtsack immer mehr zusammenfällt, bis schliesslich der grösste Theil des Fruchtwassers demselben entzogen ist. Wenn man weiterhin ein Röhrchen mit Glycerin an einem Ende durch ein Stückchen Eihaut verschliesst und dieses Ende dann in gefärbtes Wasser tauchen lässt, so wird man bald bemerken, dass sich das Glycerin zu färben beginnt und rasch an Volumen zunimmt, während das Wasser abnimmt; ein Zeichen, dass das Wasser in grösseren Mengen vom Glycerin seinem Behälter entzogen wird. $\mathrm{Zu}$ einem Controlversuche habe ich umgekehrt ein Röhrchen mit Wasser in gefärbtes Glycerin tauchen lassen. Hier blieb das Wasser vollständig klar, nahm aber an Volumen schnell ab; ein Beweis, dass es wiederum vom Glycerin stark aufgenommen wurde.

Höchst wahrscheinlich löst das Glycerin auch direct Uteruscontractionen durch Reizung der Uterus-Innenfläche aus. Was aber gerade $\mathrm{ihm}$ vor den früher gebräuchlichen Injectionsflüssigkeiten den Vorzug giebt, ist seine antiseptische Wirkung und die Eigenschaft, die Cervix geschmeidig zu machen, so dass eine schöne Blasenbildung und die Geburt verhältnissmässig leicht erfolgt. 
Diese Eigenschaften nun eröffnen dem Glycerin noch eine weitere Perspective. Denn nicht allein zur Einleitung der künstlichen Frühgeburt, nein, überall da wird man es mit Vortheil anwenden können, wo am Ende der Schwangerschaft eine möglichst schnelle Beendigung der Geburt nothwendig würde. Hierher wären alle die Zufälle zu rechnen, die das Leben der Mutter oder des Kindes bedrohen, wie z. B. Eklampsie und Blutung bei Placenta praevia. Gerade bei Placenta praevia möchte ich das Mittel unter Hinweis auf den vorhin erwähnten Fall empfehlen, wo durch Blutung und Fieber Mutter und Kind sehr bedroht waren. Durch baldige kräftige Wehen war der Muttermund in zwei Stunden so erweitert, dass durch Wendung der Frucht der Blutung ein Ziel gesetzt werden konnte.

Auch bei Wehenschwäche haben wir das Mittel zwei Mal mit Erfolg angewendet:

1. Fall. K. F., 20 Jahre alt, Ipara. Letzte Periode 9. Januar 1891.

Am 15. October 1891, Vormittags 41/2 Uhr kam Patientin mit schwachen Wehen auf den Geburtssaal. Die Wehen wurden schliesslich so schwach, dass sie nur mehr alle halbe Stunden eine Minute lang erfolgten. $10 \mathrm{Uhr}$ Vormittags Injection von etwa $80 \mathrm{~g}$ Glycerin bei dreimarkstückgrossem Muttermunde. Die Wehen wurden fast sofort kräftig und regelmässig. $11 \frac{1}{2}$ Uhr war der Muttermund vollständig erweitert. Blasensprung $11^{3} / 4 \mathrm{Uhr}$ und $12^{1} / 4$ Uhr Geburt des Kindes. Wochenbett normal. Am zehnten Tage Mutter und Kind gesund entlassen.

2. Fall. Frau M., 35 Jahre alt. Letzte Periode am 26. November 1890. 18. April 1891 erste Kindesbewegungen. Während der Schwangerschaft wenig Beschwerden. Im letzten Monate Leib sehr ausgedehnt. Schmerzen beim Sitzen.

Wehenanfang am 17. September 1891, 11 Uhr Abends. Die Wehen waren sehr schwach und selten. Dabei fingen die Kindesbewegungen, welche die Frau vorher deutlich gefïhlt hatte, an nachzulassen, die Herztöne langsamer und undeutlich zu werden. Der Muttermund, für den Finger eben durchgängig, war sehr rigid.

Das Becken war ein einfach plattes, dabei der Kindskopf unverhältnissmässig gross. Unter diesen Umständen schien es im Interesse für Mutter und Kind zu liegen, die Geburt, welche eine sehr protrahirte $\mathrm{zu}$ werden drohte, möglichst zu beschleunigen. 
Am 18. September, 2 Uhr Nachmittags intrauterine Injection von etwa $100 \mathrm{ccm}$ Glycerin. Eine Stunde später Beginn von regelmässigen, sehr kräftigen Wehen, die am 19. September, Morgens 6 Uhr ganz aufhörten, um Nachmittags gegen 3 Uhr wieder einzusetzen. Mittlerweile waren die Herztöne immer schwächer geworden und schliesslich ganz erloschen. Abends 61/2 Uhr Wendung. Da der Kopf sich auch mit der grössten Mühe nicht entwickeln liess, Perforation am nachfolgenden Kopf und Extraction desselben. Es entstand ein Dammriss III. Grades. Das Kind wog enthirnt. noch immer 14 Pfund. Das Wochenbett verlief normal.

Ein anderes Mal wurde die Wehenthätigkeit am Ende der Schwangerschaft angeregt, weil die Schwangere durch verschiedene Umstände sehr bedroht war.

Frau N., 25 Jahre alt, I para, schwächliche Person, die früher viel an Bleichsucht gelitten. Seit zehn Tagen hartnäckige Stuhlverstopfung. Grosse Dosen Calomel und öftere Einläufe, welche ihr vom behandelnden Arzte verordnet waren, hatten keine Wirkung. Starke Schmerzen und Erbrechen auch der geringsten Nahrungsmengen.

Befund am 17. October 1891, Abends: Anämische, collabirt aussehende Fran, die grosse Unruhe zeigt. Singultus, schneller kleiner Puls; kein Fieber. Leib, besonders in der Magengegend, aufgetrieben; überall auf Druck schmerzhaft. Zunge trocken bei grossem Durste. Unterer Gebärmutterabschnitt ballonartig vorgetrieben. Muttermund mit Mühe für den Finger durchgängig. Kopf vorliegend.

Die Nacht hydropathische Umschläge um den ganzen Leib. Am 18. October, 11 Uhr Morgens intrauterine Injection von etwa $100 \mathrm{ccm}$ Glycerin. Eine halbe Stunde später Beginn der Wehen, 1 Uhr Mittags Blasensprung bei vollständig erweitertem Muttermunde. Um 4 Uhr Nachmittags Geburt des Kindes, welches einen ungewöhnlich grossen Kopf hatte. Die Kopfumfänge waren: horizontaler Umfang $36 \mathrm{~cm}$, kleine Diagonalis $34 \mathrm{~cm}$ und grosse Diagonalis $41 \mathrm{~cm}$.

An der Hand der vorher angeführten Krankengeschichten sehen wir deutlich, dass wir in der intrauterinen Injection von Glycerin ein Mittel besitzen, welches den weitesten Anforderungen, die man an eine Methode, die künstliche Frühgeburt einzuleiten, stellen kann, zum mindesten sehr nahe kommt. 
228 Pelzer, Erregen d. Wehenthätigkeit durch intrauter. Inject. u. s. w.

Die regelmässigen Wehen setzen alsbald nach der Injection ein. Es erfolgt eine schöne Blasenbildung und die Geburt verhältnissmässig schnell und leicht. Dabei ist die Gefahr der Infection bei der nöthigen Vorsicht eine äusserst geringe, die der Luftembolie kaum denkbar.

\section{Nachtrag.}

Nach Schluss vorstehender Arbeit bemerkte ich in Kehrer's „Lehrbuch der operativen Geburtshülfe“" vom Jahre 1891, dass derselbe in mehreren Fällen mit Erfolg die Glycerin-Wattetamponade des. Mutterhalses angewendet hat. Eine Beschreibung der betreffenden Fälle ist mir in der Literatur nicht aufgefallen. Die Methode Kehrer's und die intrauterine Injection von Glycerin haben ja entschieden etwas Verwandtes, und es wäre vielleicht interessant, wenn durch weitere nach Kehrer's Methode behandelte Fälle die Erfahrungen in dieser Hinsicht bereichert würden.

Zuletzt erlaube ich mir noch darauf aufmerksam zu machen, dass unsere Beobachtungen schon von Juli des Jahres 1890 her datiren. 